D.T. Wickramasinghe, L. Ferrario, and G.V. Bicknell, eds.

\title{
Mass Flux and Toroidal Magnetic Field in Magnetically Driven Jets from Accretion Disks: Steady Solutions and Application to Jet/Wind in YSO
}

\author{
T. Kudoh and K. Shibata \\ National Astronomical Observatory of Japan, 2-21-1, Osawa, Mitaka, \\ Tokyo, 181, Japan
}

\begin{abstract}
We solved one-dimensional steady and axisymmetric magnetohydrodynamical (MHD) equations to study basic properties of astrophysical jets from accretion disks. We found that the mass flux of the jets depends on the poloidal magnetic field strength of the disk. The mass loss rates observed in $\mathrm{T}$ Tauri winds will constrain the physical quantities in the accretion disks.
\end{abstract}

\section{Introduction}

We study astrophysical jets from accretion disks by solving one-dimensional steady and axisymmetric MHD equations exaclty. One-dimensional means that the shape of a poloidal magnetic field line is assumed and the equation of motion perpendicular to the poloidal field line are not solved for simplicity. The simple one-dimensional solution clarifies basic physics of magnetically driven jets from accretion disks (Cao \& Spruit 1994, Kudoh \& Shibata 1995), and it helps us to understand features of time-dependent MHD jets which are performed by e.g., Shibata \& Uchida (1986) (see Kudoh, Matsumoto, \& Shibata in this proceeding).

\section{Results}

We include a thermal energy although the jet is mainly accelerated by magnetic force, so that we can obtain the mass flux of the jet. We found that the mass flux depends on the poloidal magnetic field strength in the disk when the toroidal component of the magnetic field become dominant near the disk surface, although it is independent of the magnetic field when the the poloidal component is dominant there (Fig. 1ab);

$$
\dot{M} \propto \begin{cases}\text { constant } & \left(\left|B_{\phi} / B_{p}\right|_{0} \ll 1\right) \\ B_{p 0} & \left(\left|B_{\phi} / B_{p}\right|_{0} \gg 1\right)\end{cases}
$$

where $\dot{M}$ is the mass flux of the jet, $B_{p}$ and $B_{\phi}$ are the poloidal and toroidal component of the magnetic field, and 0 denotes the value at the footpoint of the jet. When the toroidal component of the magnetic field is dominant near the disk surface, the magnetic pressure plays an important role for the total accelerations of these jets (Kudoh \& Shibata 1997). 
(a)

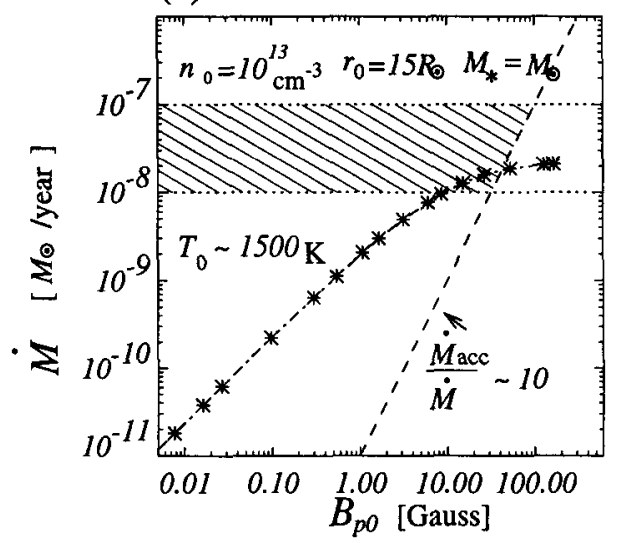

(b)

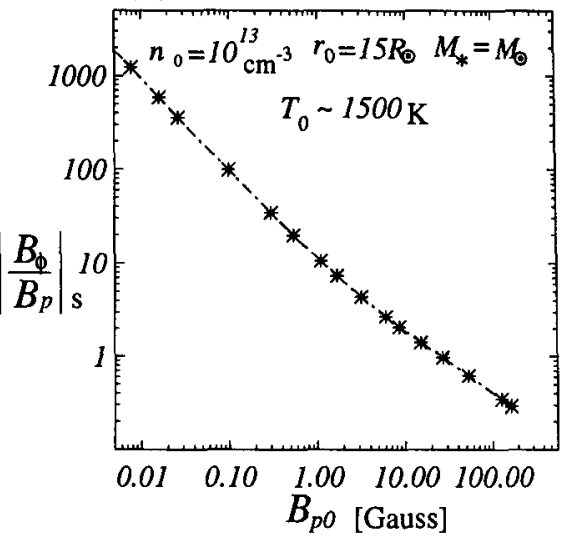

Figure 1. (a) The mass loss rates as a function of the poloidal magnetic field at the footpoint of the jet. The hatched area is consistent with observations of YSO outflows $\left(\dot{M} \sim 10^{-8}-10^{-7} M_{\odot} /\right.$ mear, and $\dot{M}_{\text {acc }} / \dot{M}<10$, where $\dot{M}_{\text {acc }}$ is an accretion rate in the disk). (b) $\left|B_{\phi} / B_{p}\right|$ at the slow point as a function of the poloidal magnetic field. (The slow point is located near the disk surface.) It is assumed that $n_{0}=10^{13}$ $\mathrm{cm}^{-3}, r_{0}=15 R_{\odot}$, and $M_{*}=M_{\odot}$, where $r_{0}$ is the radius of the footpoint of the jet, and $M_{*}$ is the mass of a central star.

\section{Application to jet/wind in YSO}

By using the mass loss rate observed in T Tauri stars $\left(\dot{M} \sim 10^{-8}-10^{-7} M_{\odot} /\right.$ year $)$, we can estimate the physical quantities in the accretion disk, such as number density $n_{0}$, temperature $T_{0}$, and poloidal component of the magnetic field $B_{p 0}$. They are estimated to be $n_{0} \sim 10^{13} \mathrm{~cm}^{-3}, T_{0} \sim 1000 \mathrm{~K}$ and $B_{p 0} \sim 10 \mathrm{G}$, for the jet which is produced at $r_{0} \sim 15 R_{\odot}$ (Fig.1), and its terminal velocity is $\sim 100 \mathrm{~km} / \mathrm{s}$ (the terminal velocities are of order of Keplerian velocity of the disk (Kudoh \& Shibata 1995)). These values are in rough agreement with those estimated by Shu et al. (1994). However, we found that the toroidal components of the magnetic fields are obtained to be the same order as the poloidal one near the disk surface $\left(\left|B_{\phi} / B_{p}\right|_{0} \sim 1\right)$ (Fig. 1b), which was not well appreciated by Shu et al. (1994).

\section{References}

Cao, X., \& Spruit, H.C. 1994, A\&A, 287, 80

Kudoh, T., Matsumoto, R., \& Shibata, K., in this proceeding

Kudoh, T., \& Shibata, K. 1995, ApJ, 452, L41

Kudoh, T., \& Shibata, K. 1997, in press, ApJ, 474, Jan.1

Shibata, K., \& Uchida, Y. 1986, PASJ, 38, 631

Shu, F. et al., 1994, ApJ, 429, 781 